\title{
A modular toolset for electrogenetics
}

Lawrence, J. M.1,2, , Yin, Y. ${ }^{3, \S}{ }^{\dagger}$, Bombelli, P.1,4, Scarampi, A. ${ }^{1}$, Storch, M.5 , Wey, L. T. ${ }^{1}$, Climent-Catala , A. ${ }^{6}$, PixCell iGEM team ${ }^{+}$, Baldwin, G. S.7 , O’Hare, D. ${ }^{3}$, Howe, C. J.1 ${ }^{1}$ Zhang, J. Z2 ${ }^{2}$ Ouldridge, T. E. ${ }^{3}$, Ledesma-Amaro, R. ${ }^{3, *}$.

+Lawrence, J. M.1,2, Yin, Y. ${ }^{3, \dagger}$, Scarampi, A. ${ }^{1}$, Beardall, W. ${ }^{3}$, Caganek, T.7,, , Chang, S.7, ,T,

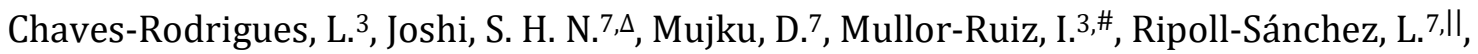
Singh, A. ${ }^{3}$

${ }^{1}$ Department of Biochemistry, University of Cambridge.

2 Yusuf Hamied Department of Chemistry, University of Cambridge.

3 Department of Bioengineering, Imperial College London.

4 Department of Environmental Science and Policy, Università degli Studi di Milano.

${ }^{5}$ London DNA Foundry, Imperial College Translation \& Innovation Hub.

6 Department of Chemistry, Imperial College London.

7 Department of Life Sciences, Imperial College London.

† Current Address: Department of Engineering Science, University of Oxford.

‡ Current Address: Department of Chemistry, University of Oxford.

ฯ Current Address: Institute of Plant and Molecular Biology, Academia Sinica.

${ }^{\Delta}$ Current Address: Department of Engineering, New York University, Abu Dhabi.

\# Current Address: Hamilton Institute, Maynooth University.

Il Current Address: Neurobiology Division, MRC Laboratory of Molecular Biology, Cambridge.

$\S$ These authors contributed equally to this work

*Corresponding author 


\begin{abstract}
Synthetic biology research and its industrial applications rely on the deterministic spatiotemporal control of gene expression. Recently, electrochemical control of gene expression has been demonstrated in electrogenetic systems (redox-responsive promoters used alongside redox inducers and an electrode), allowing for the direct integration of electronics with complex biological processes for a variety of new applications. However, the use of electrogenetic systems is limited by poor activity, tunability and standardisation. Here, we have developed a variety of genetic and electrochemical tools that facilitate the design and vastly improve the performance of electrogenetic systems. We developed a strong, unidirectional, redox-responsive promoter before deriving a mutant promoter library with a spectrum of strengths. We then constructed genetic circuits with these parts and demonstrated their activation by multiple classes of redox molecules. Finally, we demonstrated electrochemical activation of gene expression in aerobic conditions utilising a novel, modular bioelectrochemical device. This toolset provides researchers with all the elements needed to design and build optimised electrogenetic systems for specific applications.
\end{abstract}

\title{
Introduction
}

Advances in synthetic biology have allowed for the development of a variety of genetic circuit-based devices used for sensing applications(1), drug and chemical production(2) and material synthesis(3). However, deterministic regulation of these systems is limited compared to the precise system control provided by electronic circuits. Integrating the control of electronic circuits with the biocatalytic activities of cells has the potential to create reliable and affordable devices for various biomedical and industrial applications(4).

One major method used to control biological systems is the use of inducible gene expression systems, which consist of a promoter and its cognate transcription factor that can either activate or repress the expression of genes in response to external stimuli(5). These systems have been utilised in molecular biology for the study of biomolecular systems in a variety of organisms (6-9). Inducible gene expression systems also serve as key tools in synthetic biology, being used as inputs for genetic logic circuits (10), in microbial and cell-free biosensors $(11,12)$, and as control systems for CRISPR-Cas9 genome editing(13), to name but a few applications.

Whilst chemically inducible and light inducible (optogenetic) gene expression systems are the most popular, due to their strong activities, simplicity and robustness $(14,15)$, other inducible systems have been identified or engineered that respond to heat(16), magnetism(17), pressure(18), osmolarity(19), salinity(20) and gravity(21). Electrogenetics is a nascent field of research in which electrical and electrochemical stimuli are used to control biological processes, including gene expression $(4,22)$. Electrogenetic systems consist of four components: an electrode, a redox inducer, a redox-transcription factor and its cognate promoter (Fig. 1a). The electrode is held at a set potential that is used to oxidise or reduce the redox inducer, which is a cell- 
permeable electron mediator. The redox inducer in turn oxidises or reduces the redoxtranscription factor, which leads to activation or repression of gene expression from its cognate promoter.

Unlike other inducible gene expression systems, electrochemical control of gene expression allows for electronic and genetic circuits to be directly connected to one another. The ability to combine electronic controllers easily with cellular biocatalysts is expected to revolutionise bioelectronics, facilitating easy design of devices for biosensing, medical diagnostics and therapies, information processing and energy conversion(23-25). Electrogenetic systems also have high spatiotemporal control compared to other widely used gene expression systems. They can be used to provide rapid oscillatory gene expression(22), as well as spatial gradients of gene expression across cells immobilised in hydrogels(26). Already, electrogenetic systems capable of performing digital-to-biological data storage(27) and electrically-controlled in vivo insulin delivery(28) have been developed. Despite these advances, electrogenetics remains an emerging field, with the development of new systems being hindered by a lack of effective and standardised tools, whether they be electrochemical apparatus, redox inducers or genetic parts. Use of the systems has also been limited due to many of them requiring anaerobic conditions(22) or complex co-cultures(29). Oxidation of redox inducers by oxygen is common, which complicates accurate control of gene expression and can lead to the formation of cytotoxic reactive oxygen species in aerobic conditions.

In a pioneering study, Tschirhart et al. first demonstrated electronic control of gene expression in bacteria by repurposing the soxRS oxidative stress response operon of Escherichia coli(22) (Fig. 1b). In this system a gold electrode was used, with the redox inducer pyocyanin oxidising the SoxR transcription factor to activate the expression of transgenes placed downstream of the PsoxS promoter. The precise spatiotemporal control of gene expression that has been demonstrated with SoxR-PsoxS make it a desirable electrochemically inducible gene expression system $(22,26)$. However, the system has not yet been used to activate gene expression controllably in aerobic conditions due to pyocyanin's reactivity with oxygen(30). The native PsoxS promoter also exhibits a limited dynamic range (DynR, the fold-change between the maximum activity and the basal activity) when compared to chemical and optogenetic inducible system $(14,15,22)$. The lack of a PsoxS promoter library and the bidirectional nature of the promoter also prevent rational design and standardised assembly of genetic circuits $(31,32)$.

In this work, we redesigned the PsoxS promoter to facilitate stronger electrochemical induction of gene expression. A unidirectional form of the PsoxS promoter was engineered with greater dynamic range, allowing for simple construction of genetic circuits. We also created a library of PsoxS promoters with a spectrum of activities, and tested activation of genetic circuits utilising our promoters with several different classes of redox inducer. Finally, electrochemical activation of gene expression was demonstrated in aerobic conditions using a novel, modular bioelectrochemical device designed for use in electrogenetic systems. These results establish the viability of utilising electrochemically inducible gene expression in synthetic biology. The genetic and electrochemical tools we have developed (Fig. 1c), along with the standardised 
design principles used, will enable the simple construction of electrogenetic systems for diverse applications.
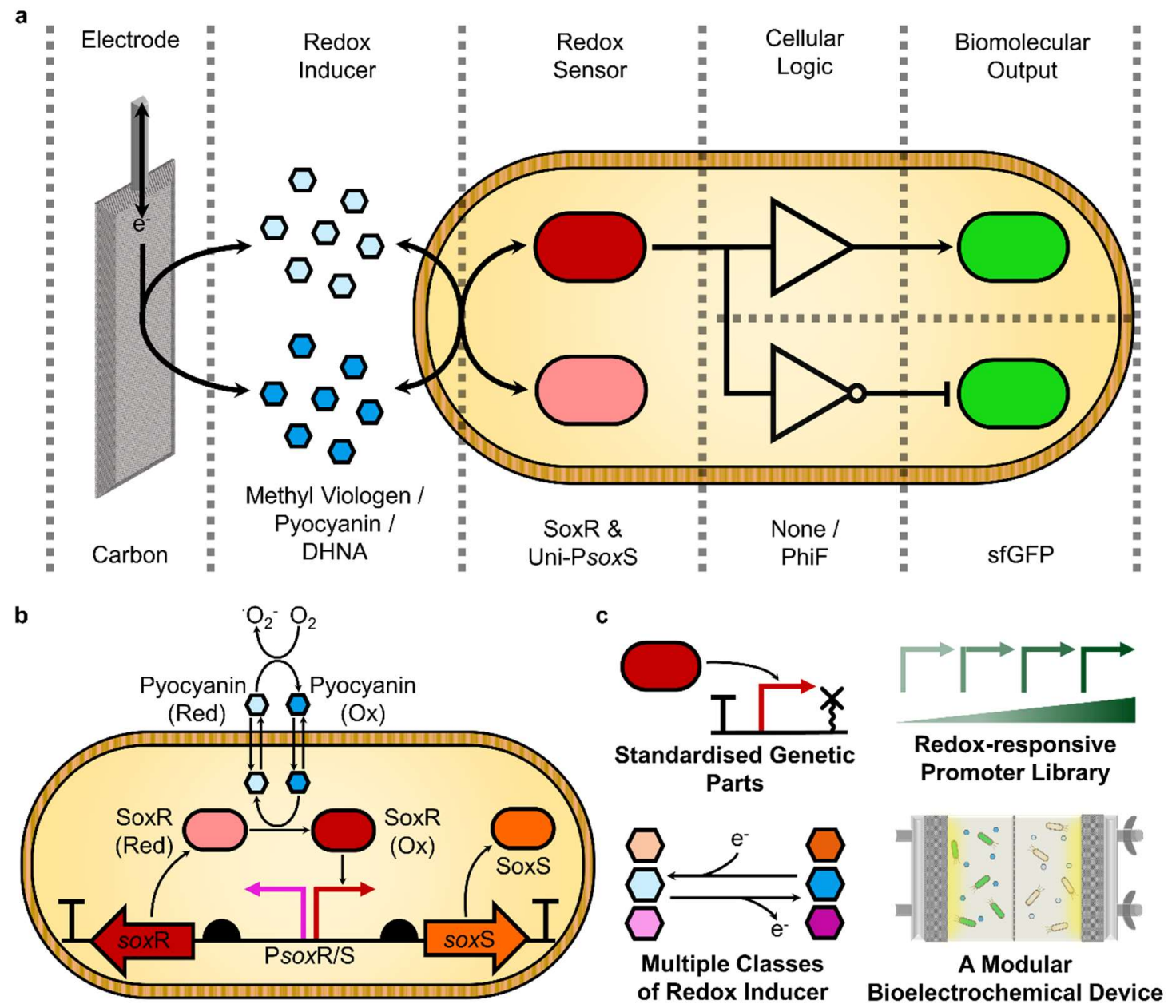

Fig. 1: Electrochemical control of gene expression in electrogenetic systems.

a, Electrogenetic systems consist of a series of electrochemical and genetic modules. An electrode controls the redox state of a redox mediator that the cell responds to with a redox-sensitive transcription factor. Depending on the cellular logic encoded by the genetic architecture used, oxidation of the transcription factor will either activate or repress expression of the chosen gene-ofinterest. The identity of specific components tested in this study are labelled beneath the schematic. b, The native soxRS oxidative stress response system of $E$. coli. SoxR is constitutively expressed from the PsoxR promoter. Oxidation of SoxR by oxidative-stress inducing redox molecules such as pyocyanin causes transcriptional activation of the PsoxS promoter. This activation leads to SoxS expression, which activates transcription of numerous genes involved in the oxidative stress response. The redox-dependency of PsoxS allows for it to be utilised for electrochemical induction of gene expression. c, An overview of the genetic and electrochemical tools developed in this study for use in electrogenetics systems. 


\section{Results}

\section{Re-designing the redox-responsive PsoxS promoter:}

The bidirectional PsoxR/S promoter lies at the core of the soxRS operon (Fig. 1b; Fig. 2a). The upstream PsoxR promoter constitutively expresses the SoxR transcription factor, the Fe-S clusters of which can be oxidised by various redox-cycling drugs $(33,34)$. SoxR binds to operator sites in the downstream PsoxR promoter so that upon its oxidation conformational changes in the protein bring these sites closer together, reducing the distance between the promoter -35 and -10 sites allowing for RNA polymerase recruitment and transcription of the gene for the SoxS regulatory protein(35).

The bidirectional nature of the PsoxR/S make it hard to employ in genetic circuits, whilst its overlapping regulatory sequences make it difficult to generate mutant variants of the promoter(36, 37) (Fig. 2a). To solve these problems, we have redesigned the PsoxR/S promoter in order to obtain a unidirectional redox-responsive PsoxS promoter. We created two variants of the native promoter: Bi-PsoxS and Uni-PsoxS. Both were designed with a standardised architecture of an upstream terminator, a spacer sequence and a downstream RiboJ sequence(38) (Fig. 2b). These genetic elements were added to reduce promoter context dependency $(39,40)$, whilst the terminator was additionally expected to remove upstream transcriptional activity and aid assembly of the promoter in multi-gene constructs. In Uni-PsoxS we additionally deleted the PsoxR -35 regulatory sequence to enhance unidirectional promoter activity. We rationalised that removing PsoxR activity would also enhance the activity of the promoter by favouring recruitment of RNA polymerase in the PsoxS direction.

We cloned native, Bi-PsoxS and Uni-PsoxS promoter variants into genetic circuits capable of screening their activities through the measurement of fluorescent protein expression with a microplate reader. Downstream and upstream promoter outputs were measured through the stationary phase expression of super-folder green fluorescent protein (sfGFP) and red fluorescent protein (RFP), respectively (Fig. 2c). Fluorescence data was normalised by cell density before, before being divided by the same value recorded from a standardised reference plasmid (Supplementary Table 3) in order to calculate the promoter output in relative promoter units (RPUs)(41) (Materials and Methods). This reference plasmid expressed reporters under the control of a J23101 promoter built with the same standardised architecture as Bi-PsoxS and UniPsoxS. Reporters were expressed under the control of strong synthetic ribosome binding sites (RBSs)(42) rather than the native RBS sequences(37) in all plasmids (Fig. $2 \mathrm{~d}$ ), and the gene circuits were assembled within a medium-high copy number vector (based on SEVA pBR322)(43) to maximise recorded promoter outputs.

E. coli DH5 $\alpha$ were transformed with either the assembled plasmids containing the promoter variant constructs (Fig. 2b-c) or a negative control plasmid consisting of an empty pBR322 vector (Supplementary Table 3). E. coli DH5 $\alpha$ contains an intact chromosomal copy of the soxRS operon constitutively expressing the SoxR transcription factor (Fig. 2c), allowing for the basal and induced activities of the PsoxS promoter variants to be measured through the addition of the redox inducer pyocyanin. A 
pyocyanin concentration of $2.5 \mu \mathrm{M}$ was selected to maximise promoter outputs without incurring significant cytotoxicity, which we measured by cell growth inhibition (Supplemental Fig. 1).

a

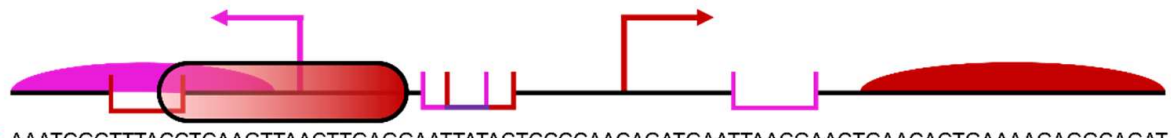

PsoxR/S AAATCGCTTTACCTCAAGTTAACTTGAGGAATTATACTCCCCAACAGATGAATTAACGAACTGAACACTGAAAAGAGGCAGAT

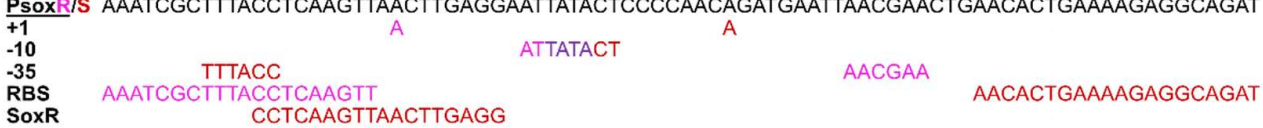$$
\text { SoxR }
$$
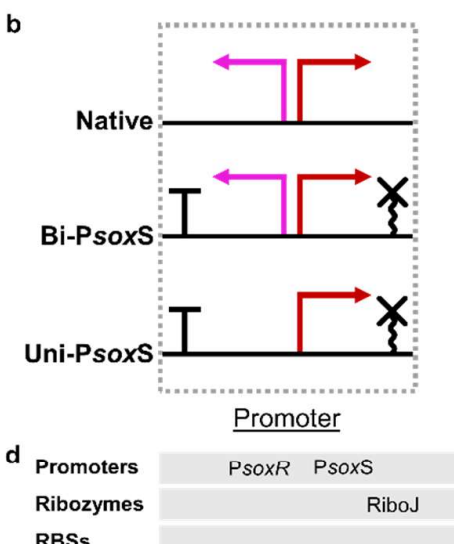

RBSs

Terminators L3S2P21

C

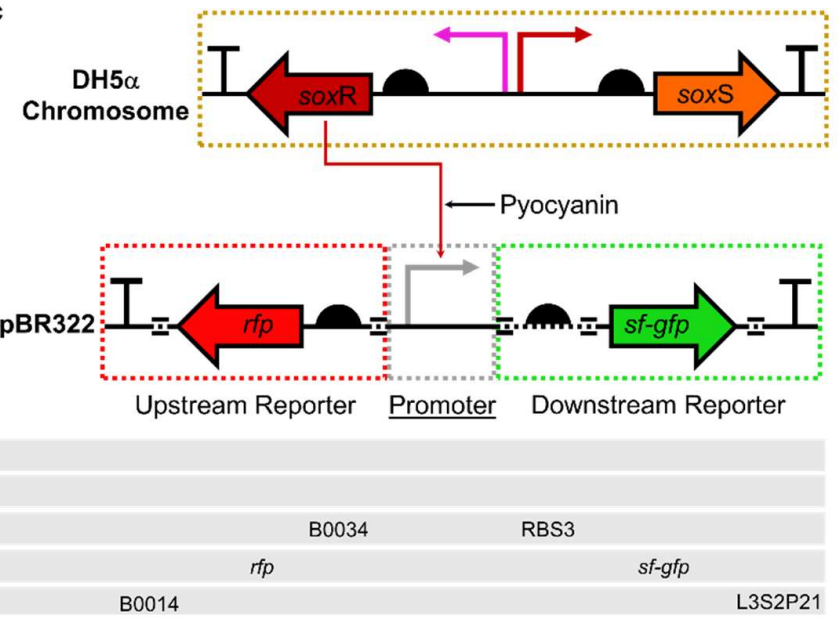

e
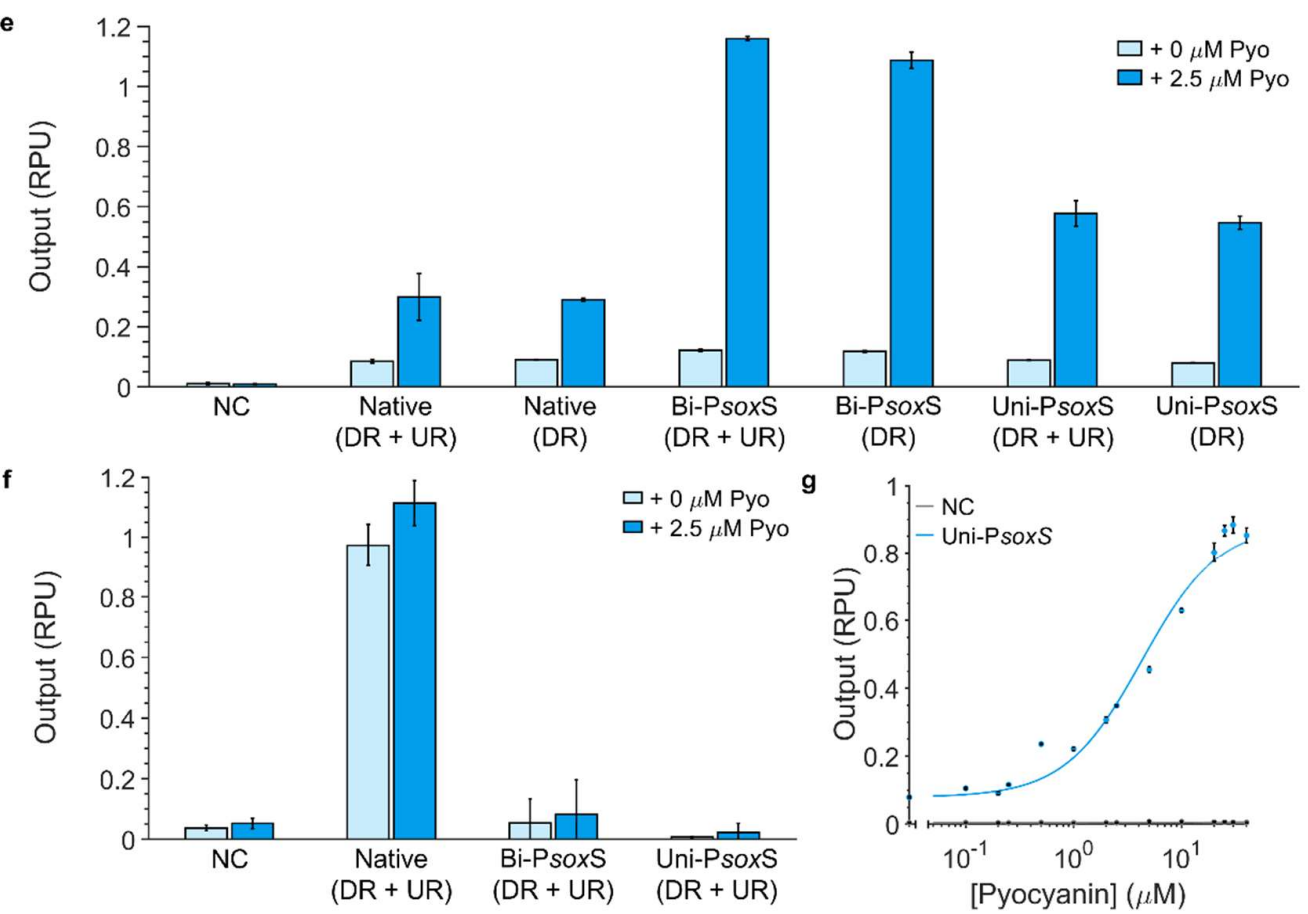

Fig. 2: Screening the activity and directionality of mutant PsoxS promoters.

a, The sequence of the native PsoxR/S promoter, with key regulatory components of PsoxR (pink), PsoxS (red) and overlapping components (purple) labelled. b, Architectures of native and engineered PsoxS promoters tested. c, Genetic circuit design for screening PsoxS promoters for upstream and downstream transcriptional activity. Circuits can consist of both upstream and downstream reporters, or just the downstream reporter. Black dotted regions of the DNA represent BASIC linkers. $\mathbf{d}$, List of 
genetic parts used. e, Downstream activity of engineered PsoxS promoters with and without $2.5 \mu \mathrm{M}$ pyocyanin. f, Upstream activity of engineered PsoxS promoters with and without $2.5 \mu \mathrm{M}$ pyocyanin. $\mathbf{g}$, The response function of the Uni-PsoxS (DR) promoter with pyocyanin. Datapoints represent the mean from three biological replicates with error bars depicting standard deviation $(n=3)$. UR, upstream reporter; DR, downstream reporter; RPU, relative promoter units.

All promoter variants displayed an induction of downstream promoter output in the presence of pyocyanin. However both mutant variants displayed a significanty enhanced fold-change between the uninduced and induced outputs compared to the native promoter (Fig. 2e). In constructs excluding the upstream reporter, the difference between the induced and uninduced downstream promoter outputs was 3.22-fold for the native promoter, compared to 9.20-fold and 6.86-fold for the Bi-PsoxS and Uni-PsoxS variants respectively. Both mutant promoter variants we designed also lacked upstream promoter activity (Fig. 2f), facilitating their use in multi-gene devices without generating unwanted transcripts.

Whilst the fold-change of the Bi-PsoxS promoter was higher than for the Uni-PsoxS promoter, the latter has a significant $32.6 \%$ reduction in basal activity in the absence of pyocyanin than the former (Fig. 2e). Consequently, the Uni-PsoxS variant was selected for use in future constructs. The downstream activity of the promoter was recorded in response to differing concentrations of pyocyanin and was fitted with a response function(14) (Fig. 2g; Supplementary Table 1). Response function curve fitting yielded a curve with cooperativity $(n)$ of 1.22 and a sensitivity $(K)$ of $4.29 \mu \mathrm{M}$. This $K$ value is comparable to some of the highest performing chemically inducible gene expression systems(14).

Previous research has suggested that the PsoxR promoter is repressed by SoxR(44). Furthermore, PsoxS deletions in the spacer region between the -35 and -10 sites have been shown to convert the promoter from an activator to a repressor in certain strains of E. coli(45). With this in mind, we designed a unidirectional PsoxR promoter variant (Uni-PsoxR), as well as variants of the Uni-PsoxS promoters with deletions between the 35 and -10 sites with the aim of creating promoters that are repressed rather than activated in the presence of pyocyanin. None of these exhibited significant fluorescence differences when pyocyanin was present (Supplementary Fig. 2) and were therefore not functional repressors in E. coli DH5a.

\section{Development of a redox-responsive promoter library:}

The deterministic design of complex genetic circuits requires accurate control of gene expression. Because of this, numerous promoter libraries have been generated for $E$. coli, including for both constitutive(41) and chemically inducible(46) promoters, allowing researchers to tune the expression of genes deterministically within genetic circuits. Here, we have developed a comparable library for Uni-PsoxS through mutagenesis of the -35 and -10 sites. We selected three -35 sites (termed A-C) and four 10 sites (termed a-d) to test (Fig. 3a) which we expected to provide a range of promoter activities $(46,47)$. The terminal cytosine nucleotides of the -35 site involved in SoxR binding were conserved in all variants(36). Uni-PsoxS variants with different 
combinations of -35 and -10 sites were screened with the previously described downstream reporter construct (Fig. 2c), using a pyocyanin concentration of $10 \mu \mathrm{M}$ to induce gene expression. This high concentration was selected to ensure the response of any promoters with low sensitivity would be detected (Fig. 2f). Both the uninduced and induced outputs were measured, with the fold change between them being calculated to estimate the dynamic range. However, it is important to note that some promoters may have lower sensitivities and not be fully induced in the presence of $10 \mu \mathrm{M}$ pyocyanin, meaning these fold changes may not be an accurate reflection of the dynamic range.

A range of outputs were observed amongst the Uni-PsoxS mutants (Fig. 3b-d;

Supplementary Fig. 3). Whilst no mutations had enhanced fold-change relative to unmutated Uni-PsoxS, all mutants were inducible and had altered outputs. Some promoters with -10 sites $a$ and $b$ had significant reduction in both the basal and induced output of the promoter, making them preferable for the expression of toxic genes. Promoter mutants Bd, Cc and Cd all had significantly higher basal and induced outputs, with mutant Cd providing the strongest output of any induced promoter at 1.21 RPU, which is comparable to the widely used pTet and pLac chemically inducible promoters(41).
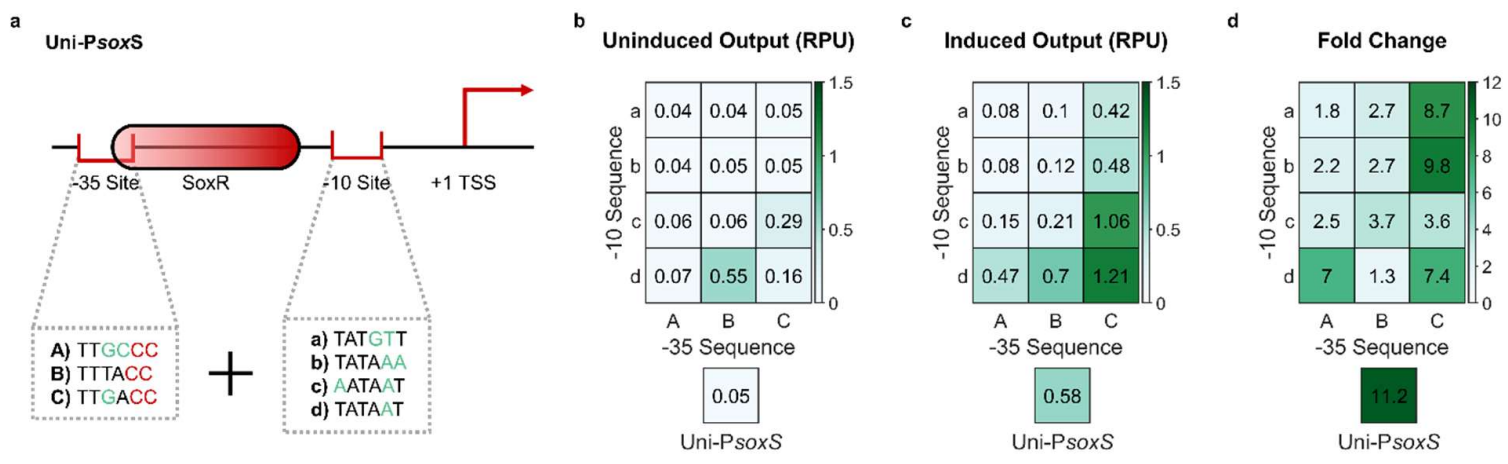

Fig. 3: A Uni-PsoxS promoter library.

a, Three -35 and four -10 site mutations were screened for tuning Uni-PsoxS activity. -35 site mutations conserved nucleotides involved in SoxR binding. Promoters were tested using the genetic circuit in Fig. 2c. b, OFF activity of the promoter library in the absence of pyocyanin. c, ON activity of the promoter library in the presence of $10 \mu \mathrm{M}$ pyocyanin. $\mathbf{d}$, Fold change between ON and OFF activities of the promoter library. Datapoints represent the mean from three biological replicates.

\section{Enhancement and modulation of electrogenetic circuit responses:}

Whilst the Uni-PsoxS promoter is functional in E. coli DH5 $\alpha$ due to its genomic copy of the soxRS(48), the low level of SoxR expression from the PsoxR promoter (Supplemental Fig. 2) is not optimised for recombinant protein expression. In order to overcome this low SoxR expression we assembled genetic circuits that not only expressed sfGFP under the control of Uni-PsoxS in a "output cassette", but also contained a "sensor cassette" in which SoxR was expressed by a constitutive promoter (Fig. 4a). We developed two versions of these "activator" genetic circuits (Act105 and Act106) with SoxR expression being controlled by a different constitutive promoter in each (Fig. 4b). These constitutive promoters were created using the same standardised promoter design as Uni-PsoxS (Fig. 2b)(38). We tested activator circuits in both DH5 $\alpha$ and DJ901, a strain 
that contains a chromosomal deletion of the soxRS operon(49). This deletion prevents the strain from expressing antioxidant proteins (44) and multi-drug efflux pumps (50), which we expected to enhance gene expression induction by pyocyanin, albeit at the expense of increased cytotoxicity(22).

Act105 and Act106 were screened for output in the absence and presence of $2.5 \mu \mathrm{M}$ pyocyanin. A higher concentration was not used due to the susceptibility of DJ901 to oxidative stress. A negative control construct was also tested (Supplementary Table 3). All circuits demonstrated an enhanced output relative to the negative control, with circuits in DJ901 outperforming those in DH5a (Fig. 4c). Act106 (DJ901) had the best performance, with the highest induced output of 2.22 RPU with 26.3-fold activation being achieved upon addition of pyocyanin, both over twice that observed with UniPsoxS alone in DH5 $\alpha$ (Fig. 2e). Interestingly, Act105 and Act106 in DH5 $\alpha$ did not provide improved performance relative to Uni-PsoxS alone in DH5 $\alpha$. Furthermore, whilst the J23106 promoter used in Act106 is much stronger than the J23105 promoter used in Act105 (http://parts.igem.org), the two circuits had comparable outputs. This suggests the activity of activator circuits in DH5 $\alpha$ is relatively insensitive to SoxR expression.

Genetic circuit design also allows researchers to modulate the activity of inducible gene expression systems using logic gates. These systems can be simple NOT gates, also known as inverters, which simply invert the signal (change an activator to a repressor and vice versa) or can be more complex logic gates that integrate the signals from multiple input promoters into a single output(31). We constructed a second set of genetic circuits, which were similar in design to the activator circuits but included a "logic cassette" in addition to output and sensor cassettes (Fig. 4d). This logic cassette consisted of a PhlF repressor(14) being expressed under the control of Uni-pSoxS, with sfGFP being expressed from its cognate PphlF promoter (Fig. 4e). This logic cassette is a NOT gate, allowing us to repress rather than activate Uni-PsoxS activity in response to an oxidised redox inducer such as pyocyanin. We therefore termed these "inverter" circuits.

Inverter circuits were tested in the same manner as the activator circuits. The negative control construct consisted solely of the logic and output cassettes, but with the UniPsoxS promoter in the logic cassette replaced with a constitutive promoter. All inverter circuits exhibited a repression of output in the presence of pyocyanin, but to differing extents (Fig. 4f). The outputs of both circuits in DJ901 were very low, with the uninduced state having an output comparable to the basal activity of the activator circuits in the same strain. By comparison, the inverter circuit outputs in DH5 $\alpha$ were significantly higher. Whilst this could partially be attributed to the increased strength of Uni-PsoxS output in DJ901, the reduced output of the negative control in this strain also suggests a differential activity of PphlF output between the two strains. As well as the soxRS deletion in DJ901, DH5 $\alpha$ also contains numerous genomic deletions not found in DJ901 which could contribute to this effect $(48,49)$. Inv106 in DH5 $\alpha$ produced the best response, with the highest uninduced output of 0.89 RPU, and a 5.69-fold repression being achieved upon addition of pyocyanin. By successfully demonstrating conversion of the SoxR-PsoxS system into a repressor we have expanded the applications of electrogenetic systems that utilise it. 
bioRxiv preprint doi: https://doi.org/10.1101/2021.09.10.459750; this version posted September 11, 2021. The copyright holder for this preprint (which was not certified by peer review) is the author/funder, who has granted bioRxiv a license to display the preprint in perpetuity. It is made available under aCC-BY-NC-ND 4.0 International license.

a

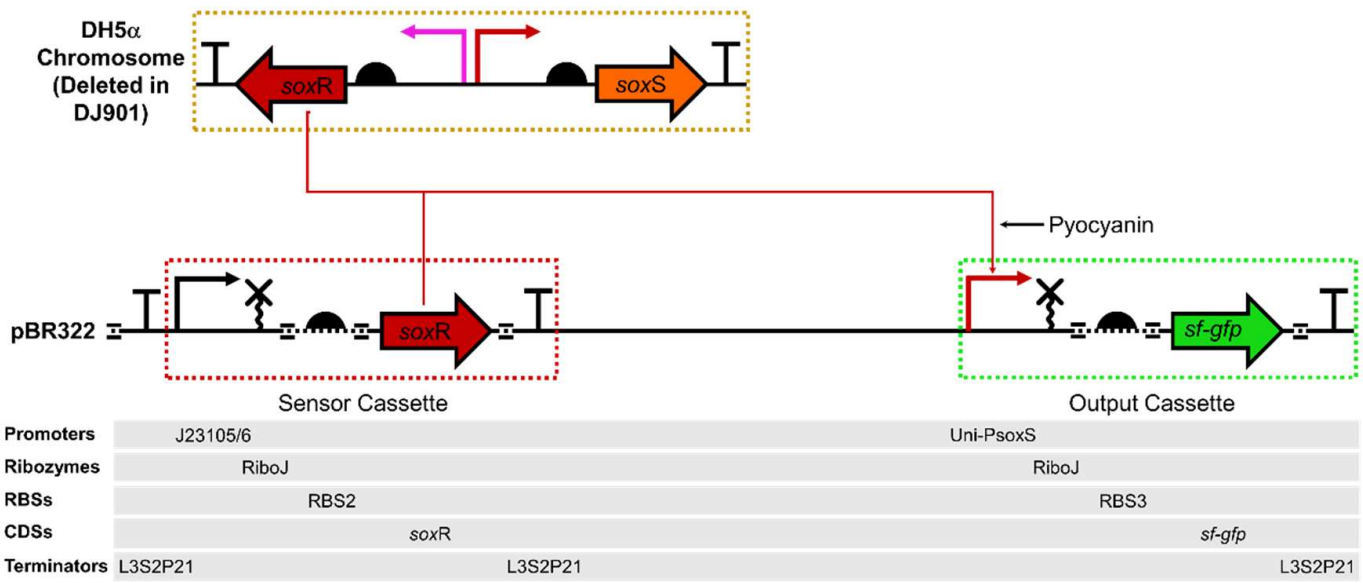

c
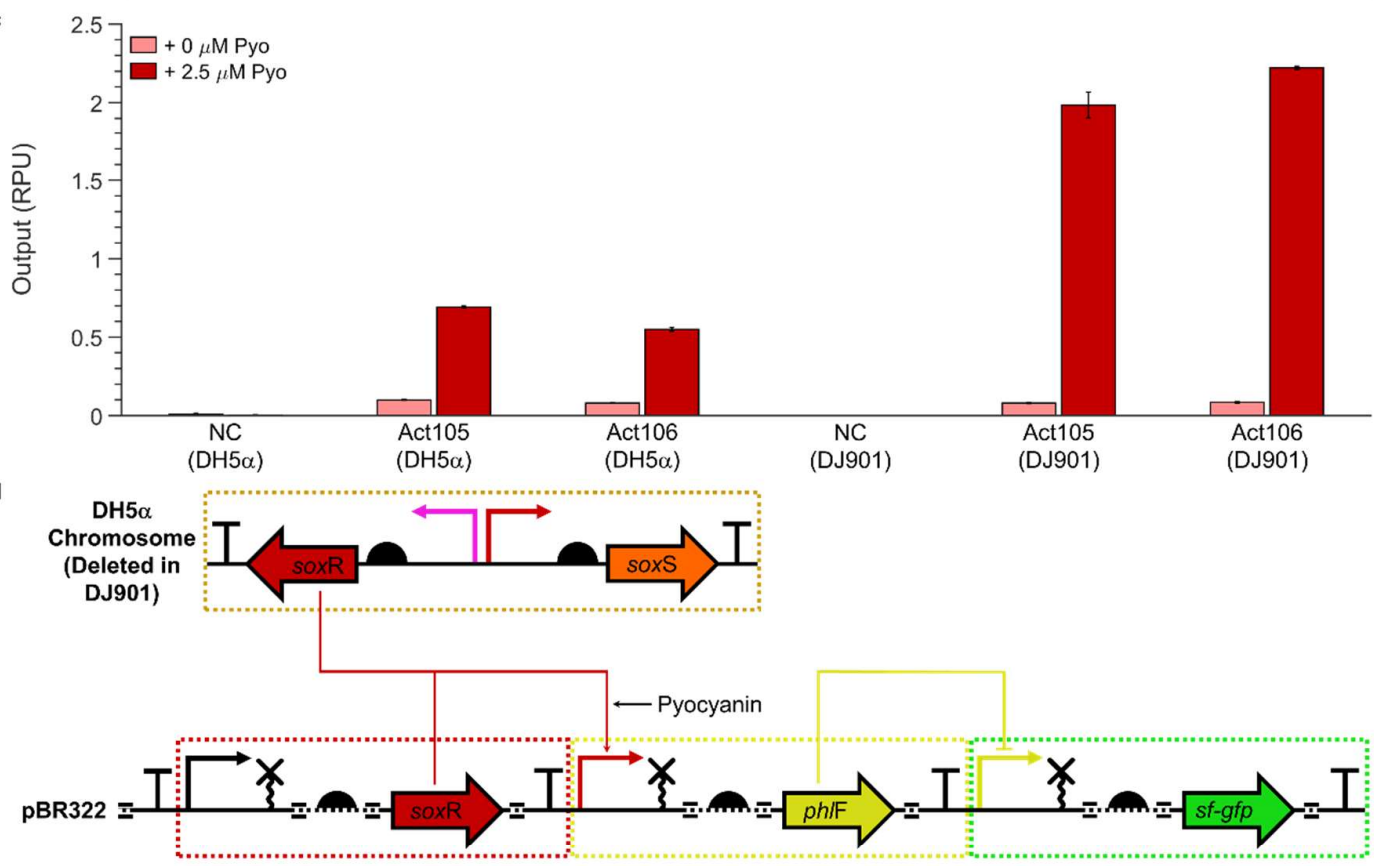

Sensor Cassette

Logic Cassette

Output Cassette

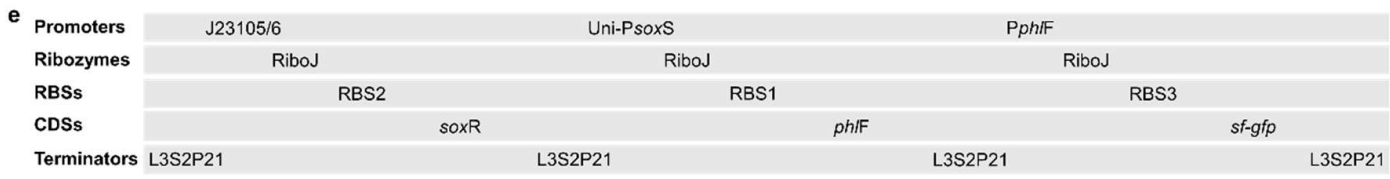

f

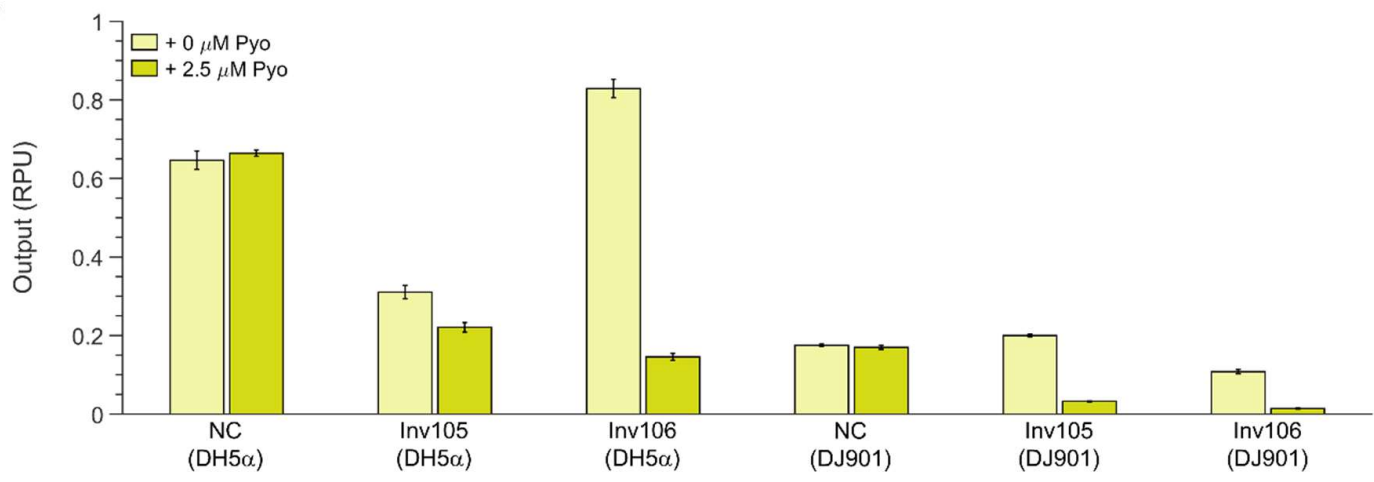


Fig 4: Performance of electrogenetic circuits in different $E$. coli strains.

a, Genetic circuit design for the activator device. A sensor cassette constitutively expressed SoxR, which, upon oxidation by pyocyanin, activates Uni-PsoxS gene expression in the output cassette. In $\mathrm{DH} 5 \alpha$ the soxRS operon is found on the chromosome, whilst it is missing in the DJ901 deletion mutant. b, List of genetic parts used in the activator device. c, Activity of activator circuit with and without $2.5 \mu \mathrm{M}$ pyocyanin. SoxR expression is controlled by J23105 in Act105 and J23106 in Act106. d, Genetic circuit design for the inverter circuit. An additional logic cassette is incorporated so that SoxR oxidation by pyocyanin activates expression of PhIF which in turn represses gene expression of the output cassette. DAPG is a chemical inducer of the PhIF-pPh/F repression system. e, List of genetic parts used in the inverter circuit. f, Activity of inverter circuit with and without $2.5 \mu \mathrm{M}$ pyocyanin. SoxR expression is controlled by J23105 in Inv105 and J23106 in Inv106. Datapoints represent the mean from three biological replicates with error bars depicting standard deviation $(n=$ 3). NC, negative control.

\section{Screening for SoxR Redox Inducers:}

When designing an electrogenetic system the choice of redox inducer is an important consideration. The membrane permeability of redox inducers will differ between organisms, whilst their midpoint potential and electron transfer chemistry will determine their effectiveness as well as their propensity to partake in cytotoxic or deleterious side reactions such as oxygen reactivity(30). Many organisms are also capable of synthesising their own redox inducers, either naturally(25) or through genetic engineering(30), which can allow for electrogenetic systems to be created without the need for exogenous redox inducers.

The ability of SoxR to be oxidised by different classes of redox inducers provides researchers with flexibility when designing electrogenetic systems(34). We demonstrated this flexibility by testing the response of our activator and inverter electrogenetic circuits with five different redox inducers: pyocyanin (a natural phenazine), methyl viologen (a synthetic viologen), DHNA (a natural quinone), riboflavin (a natural flavin) and hydrogen peroxide (Fig. 5g). All of these acted as redox inducers with the Act106 (DJ901) electrogenetic circuit apart from riboflavin and hydrogen peroxide (Supplementary Fig. 5a-c; Supplementary Fig. 4-5). Previous research has demonstrated that $E$. coli lacks riboflavin transporters(51), which could explain its lack of induction. The lack of induction by hydrogen peroxide supports previous research that SoxR primarily responds to superoxide-generating, redoxcycling drugs (52). The instability of superoxide made it hard to test the response of circuits to superoxide alone, however, previous results have shown that E. coli SoxR is relatively insensitive to oxidation by superoxide $(53,54)$.

Response function curves with different redox inducers show pyocyanin provided the highest DynR of 46.06 for Act106 (DJ901) and 49.97 for Inv106 (DH5- $\alpha$ ) (Supplementary Table 1). These values are comparable with the best performing chemically(14) and optogenetic(15) inducible gene expression systems available. The maximum output for Act106 (DJ901) was also $4.82 \mathrm{RPU}$, which is larger than any of the high-performance, plasmid-based, "Marionette" chemically inducible gene expression systems that were produced by directed evolution(14). By comparison methyl viologen and DHNA provide a lower, but still workable DynR to pyocyanin (Fig. 5a-c; 
Supplementary Table 1). This improved ability of phenazines to activate the SoxR-PsoxS system in comparison to viologens and quinones has been reported previously(54).

Cytotoxicity of the redox inducers varied (Supplementary Fig. 6). Methyl viologen was the most cytotoxic inducer, providing significant growth inhibition to both Act106 (DJ901) and Inv105 (DH5 $\alpha$ ) from concentrations of $1 \mu \mathrm{M}$. This toxicity is attributable to both its superoxide generating ability and its synthetic nature preventing it from being degraded by cells(55). As expected, pyocyanin was much more cytotoxic to Act106 (DJ901) due to the deletion of the entire soxRS operon in the strain(49), with cell growth being almost completely inhibited at $10 \mu \mathrm{M}$ with Inv106 (DH5 $\alpha$ ) exhibiting a $<20 \%$ growth inhibition at the same concentration. DHNA interestingly was only significantly cytotoxic to Inv106 (DH5 $\alpha$ ), which can likely be attributed to differences in the resistance of their respective background strains.

We also tested the performance and cytotoxicity of the Inv106 (DH5- $\alpha$ ) in M9 minimal media using pyocyanin as a redox inducer, as previous results have suggested minimal media may provide improved fold-changes with the SoxR-PsoxS system(22)

(Supplementary Fig. 7a; Supplementary Table 1). Whilst the value of $K$ is much smaller in this condition $(0.09 \mu \mathrm{M}$ in $\mathrm{M} 9 \mathrm{vs} 0.57 \mu \mathrm{M}$ in LB), this improved sensitivity is achieved at the expense of a reduced DynR (49.97 in LB vs 38.15 in M9). Little difference in the cytotoxicity of pyocyanin in the two media were observed (Supplementary Fig. 6e \& 7b).

Cyclic voltammetry was performed on the successful redox inducers pyocyanin, methyl viologen and DHNA to determine better their electrochemical properties and the mechanism by which they oxidise SoxR (Fig. $5 \mathrm{~d}$-f). Midpoint potentials ( $\mathrm{E}_{\mathrm{m}}$ ), the potential at which a $50 \%$ of the inducer is expected to be oxidised and $50 \%$ reduced, were determined from these voltammograms (Fig. $5 \mathrm{~g}$ ). If an $\mathrm{E}_{\mathrm{m}}$ value of an inducer is more positive than the $\mathrm{E}_{\mathrm{m}}$ of SoxR at $-320 \mathrm{mV}$ vs SHE ( $\mathrm{mV}$ henceforth) when bound to DNA (33), then that redox inducer is theoretically capable of directly oxidising the transcription factor. The $-5 \mathrm{mV} \mathrm{Em}_{\mathrm{m}}$ of pyocyanin and $+205 \mathrm{mV} \mathrm{Em}_{\mathrm{m}}$ of DHNA suggest they are both capable of directly oxidising SoxR and activating PsoxS expression in the process. The $-615 \mathrm{mV} \mathrm{Em}$ of methyl viologen demonstrates that it is incapable of directly oxidising SoxR and must therefore activate PsoxS expression by an indirect mechanism, as has been posited by previous studies(54).

Voltammograms recorded in air (aerobic) and under $\mathrm{N}_{2}$ purging (anaerobic) conditions were also compared to determine the extent of oxygen reduction by these redox inducers. The relative insensitivity of SoxR to superoxide $(33,54)$ and hydrogen peroxide(52) means that oxygen reduction by redox inducers is not expected to activate expression of genes downstream of PsoxS but will instead limit the ability for the redox inducer to be fully reduced by an electrode. Whilst cyclic voltammograms of DHNA showed no shift in current between scans recorded in air and under $\mathrm{N}_{2}$ purging, voltammograms of pyocyanin and methyl viologen displayed diminished oxidation (forward) peaks and altered reduction (backwards) peaks in air (Fig. 5d-f). This matches previous literature which describes the ability of pyocyanin and methyl viologen to generate reactive oxygen species(56). Current shifts were also observed in voltammograms recorded in LB in the absence of redox inducers, with a distinctive 
reduction peak being observed at $-439 \mathrm{mV}$ vs SHE (Supplementary Fig. 8), suggesting this is the peak of oxygen reduction.
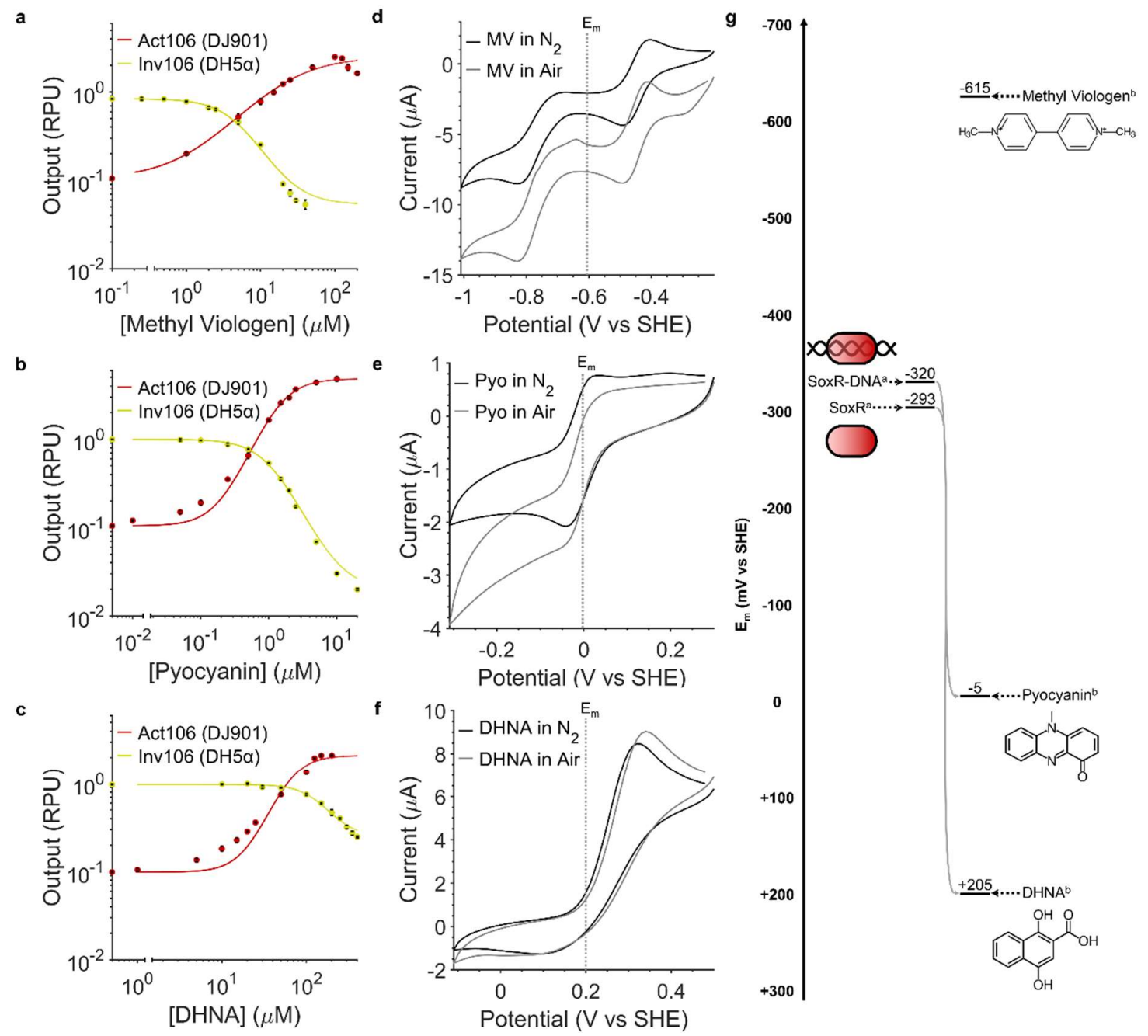

Fig 5: Electrogenetic circuit activation by diverse redox inducers.

a-c The response function of activator Act106 (DJ901) and inverter Inv105 (DH5a) circuits to methyl viologen, pyocyanin and DHNA. Datapoints represent the mean from three biological replicates with error bars depicting standard deviation $(n=3)$. d-f, Cyclic voltammograms of methyl viologen, pyocyanin and DHNA recorded in aerobic (grey lines) and anaerobic (black line) conditions. LB media was used as electrolyte with $1 \mathrm{mM}$ methyl viologen, $100 \mu \mathrm{M}$ of pyocyanin or $1 \mathrm{mM}$ of DHNA. The third cyclic voltammetry scan was recorded for each using a scan rate of $10 \mathrm{mV} \mathrm{s}^{-1}$. Voltammograms were recorded both in air and in electrolyte purged with $\mathrm{N}_{2}$ gas for 30 mins (to remove oxygen) before measurements were recorded with an $\mathrm{N}_{2}$ gas stream being maintained in the headspace. $g$, Chemical structures and redox midpoint potentials $\left(E_{m}\right)$ of redox inducers methyl viologen, pyocyanin and DHNA. Grey arrows indicate the possibility of direct oxidation of SoxR by compounds. $E_{m}$ values from ${ }^{a}$ Kobayashi, Fujikawa \& Kozawa(33) and ${ }^{b}$ this study. Pyo, Pyocyanin.

With these results we demonstrate the activation of the SoxR-PsoxS system by various classes of redox inducers across a wide range of Em values. Phenazines like pyocyanin and quinones like DHNA have an additional advantage in that they can be naturally 
synthesised. E. coli naturally synthesises DHNA(57) and has also been genetic engineered to produce pyocyanin using genes from the phz operon of Pseudomonas(58). Other electrogenic bacteria are also known to produce a variety of other electron mediators which could also act as redox inducers(25).

\section{Electrochemical Control of Gene Expression under Aerobic Conditions:}

Previous electrogenetic systems have utilised bespoke bioelectrochemical cells and devices in order to perform electrochemical control of gene expression (22, 26-29). Whilst functional, these $a d$ hoc approaches lack standardisation, preventing their use in different electrogenetic systems as well as hindering reproducibility between research groups. We have therefore assembled a bioelectrochemical device specifically designed for use in electrogenetic systems.

This device consists of a stack of acrylic blocks and polydimethylsiloxane (PDMS) gaskets with cylindrical cavities for fastening with stainless-steel screws. Electrically conductive materials serve as working and counter electrode materials with solid acrylic blocks placed at each end of the device. A Nafion ${ }^{\circledR}$ membrane placed at the middle of the stack delimits the working and counter chambers, with magnetic stir bars providing mixing in each chamber (Fig. 6a; Supplementary Fig. 9). The modular nature of the device allows for it to be reconfigured for use with different electrode materials or chamber volumes, meaning the device can be optimised for different applications. The system can be operated in 2- or 3- or 4-electrode mode by introducing one or more reference electrodes. In this study a 3-electrode set-up was used with carbon paper working and counter electrodes and $\mathrm{Ag} / \mathrm{AgCl}$ reference electrode, with each chamber having a volume of $30 \mathrm{~mL}$.

With this device we performed electrochemical activation of gene expression in aerobic conditions, utilising pyocyanin as a redox inducer due to its maximal activation of the SoxR-PsoxS system (Fig. 5b), lack of cytotoxicity with Inv106 (DH5 $\alpha$ ) (Supplementary Fig. 6e), and its previously recorded long-term stability in bioelectrochemical systems(30). Inv106 (DH5 $\alpha$ ) cells (Fig. 4d) were grown in both chambers of the device in LB electrolyte supplemented with $10 \mu \mathrm{M}$ pyocyanin under a pyocyanin-reducing applied bias potential of $-300 \mathrm{mV}$ vs SHE at the working electrode. If the rate of pyocyanin reduction by the working electrode exceeds the rate of pyocyanin oxidation by oxygen, sfGFP expression is activated in cells within the working chamber by maintaining SoxR in a more reduced state. Alternatively, in the counter chamber pyocyanin should be maintained in an oxidised state by the electrode and oxygen, leading to SoxR activation and suppression of sfGFP expression (Fig. 6b).

Under these conditions Inv106 (DH5 $\alpha$ ) exhibited a 2.27-fold increase in sfGFP expression in the working chamber relative to the counter chamber, whereas the corresponding negative control (Supplementary Table 3) construct exhibited no significant difference in sfGFP expression between the two chambers (Fig. 6c; Supplementary Fig. 10). This fold-change closely matches those reported for a different E. coli electrogenetic system in aerobic conditions(29), despite the SoxR-PsoxS here 
allowing for a much simpler electrogenetic system without the use of specialised goldbinding strains, a co-culture or minimal media.

a
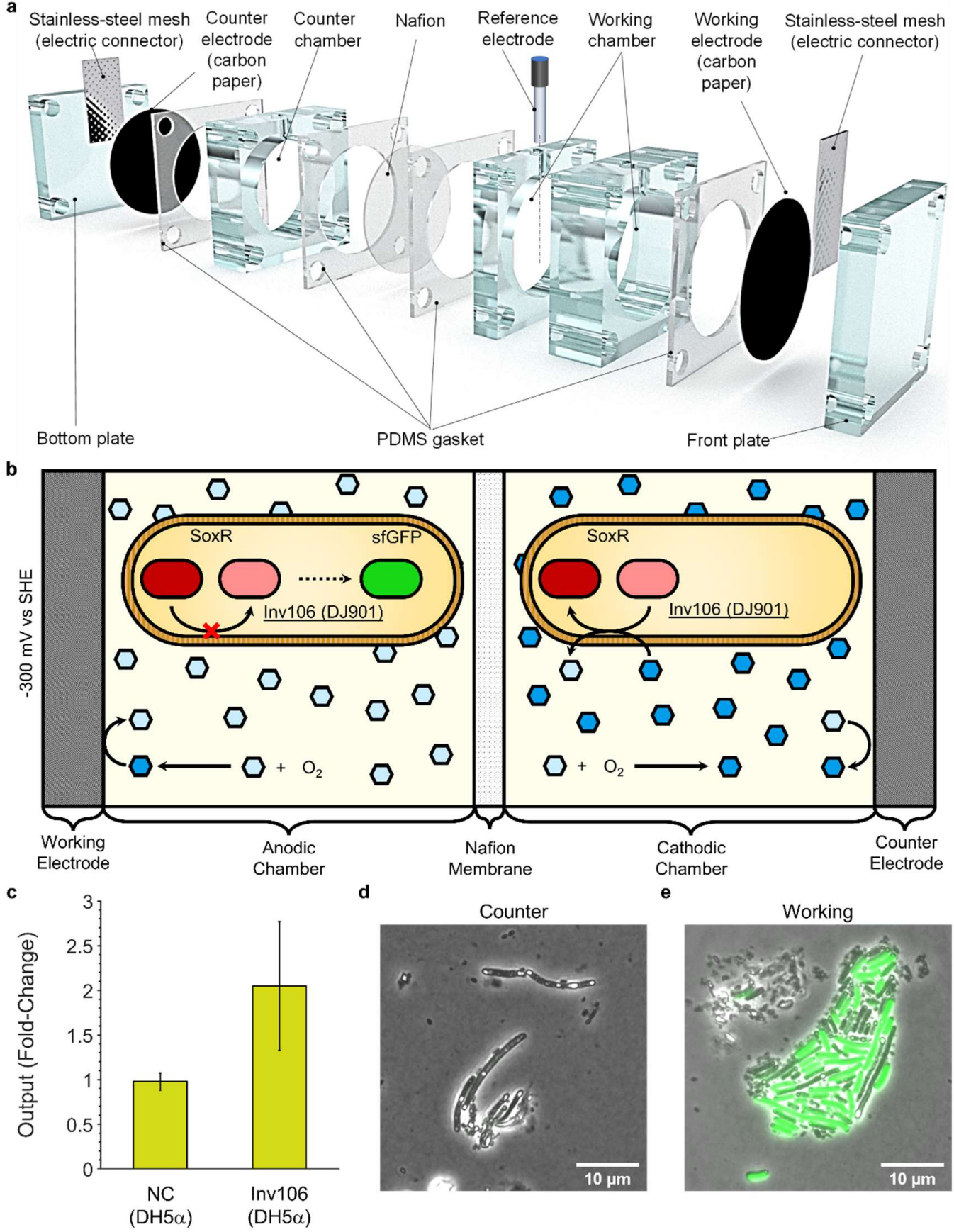

Fig 6: Electrochemical Activation of Gene Expression in Aerobic Conditions.

a, Diagram of the modular bioelectrochemical device developed for performing electrochemical induction of gene expression. $\mathbf{b}$, Schematic of device operation for electrochemical activation of gene expression. The working electrode was held at a potential of $-300 \mathrm{mV}$ vs SHE in order to reduce pyocyanin, preventing oxidation of SoxR in Inv106 (DH5a) cells and thereby activating expression of sfGFP in the anodic chamber. c, Gene expression change in cells between the working and counter chambers measured as a fold-change. Datapoints represent the mean from three biological replicates with error bars depicting standard deviation $(n=3)$. d-e, Confocal fluorescence micrographs of Inv106 
(DH5a) grown in the cathodic and anodic chambers, then immobilised on agarose pads during imaging. sfGFP fluorescence $\left(\lambda_{\text {excitation }}=490 \mathrm{~nm}\right)$ in green is overlaid on brightfield images. $\mathrm{NC}$, negative control.

The fold-change observed for electrochemical induction of Inv106 (DH5 $\alpha$ ) was much smaller than that recorded in dose response measurements (Fig. 5f), suggesting that complete reduction of pyocyanin in the anodic chamber was not achieved. Confocal fluorescence microscopy of Inv106 (DH5 $\alpha$ ) cells from the anodic and cathodic chamber also revealed that whilst cells in the anodic chamber clearly exhibited much higher sfGFP fluorescence, some cells appeared to have reduced fluorescence, suggesting certain heterogeneity within the population (Fig. 6d-e; Supplementary Fig. 11).

Regardless, these results demonstrate the first example of electrochemical activation of gene expression in reducing conditions, as well as a proof-of-concept for electrochemical control of gene expression using the SoxR-PsoxS system in aerobic conditions.

\section{Discussion}

Chemical and optogenetic inducible gene expression systems have been widely applied in molecular biology research as well as being used in various biotechnological devices. Whilst providing effective spatiotemporal control $(22,26)$ and improved integration in bioelectronic devices(4), use of electrochemical inducible gene expression systems has been limited due to their reduced performance and dependence on anaerobic conditions or co-cultures. Our results demonstrate significant improvements to the performance of the SoxR-PsoxS electrochemically inducible gene expression system, providing biological responses comparable to commonly used chemically inducible systems such as the AraC and LasR systems(46). A library of redox-responsive promoters allows for rational design of electrogenetic circuits(31), and a proof-ofconcept for electrochemical gene activation in aerobic conditions is also presented. These promoters can be used to improve the previously developed eCRISPR system for multiplexed control of expression of multiple genes (25), as well as facilitating the construction of complex multi-layered logic devices(59).

Future research should focus on the improvement of the electrochemical set-up used, as this appears to be the limiting factor in device performance. Optimal performance will be achieved with a device that can provide bulk oxidation or reduction of a stable electron mediator. The modular bioelectrochemical device presented allows for electrodes of different structures and materials to be tested, such as the various biocompatible carbon-based electrodes suitable that have previously been developed for microbial fuel cells(60). Identification of suitable redox inducers that exhibit cell permeability, long term stability, low cytotoxicity, minimal oxygen reactivity and the ability to activate the SoxR-PsoxS system strongly would provide further improvement of the device. Electrochemical limitations could also be overcome through the use of quorum sensing systems and positive feedback circuits to propagate gene expression throughout a culture $(22,26)$. Bacteria could also be engineered to express enzymes that biosynthesise redox inducers; E. coli and Pseudomonas species have previously been 
engineered to produce non-endogenous phenazines ${ }^{65,(61) . ~ G e n o m i c ~ i n t e g r a t i o n ~ o f ~}$ electrogenetic constructs will also prevent the requirement for antibiotics which are prone to electrochemical degradation(62).

The electrogenetic devices presented here can be easily adapted and integrated for use in a variety of bioelectronics, including medical and environmental biosensors (4). The demonstration of electrochemical control of gene expression in relatively large volume cultures (Fig. 6c) also suggests an application in large bioreactors, where low light penetration prevents the use of optogenetic systems. The SoxR-PsoxS system is also conserved across a diverse range of bacteria(63), with electrochemically inducible gene expression with the system being demonstrated in both E. coli and Salmonella enterica(26). Alternative redox-sensing transcription factors to SoxR have been identified in plants(64) and animals(65). The standard electrogenetic device design we have described here (Fig. 1a) can be applied to these different organisms. Furthermore, the use of the BASIC assembly standard in this study facilitates easy automation of electrogenetic circuit assembly(38), aiding the optimisation of new electrogenetic systems.

The lack of available tools for constructing electrogenetic systems has severely limited their development. The optimised toolset we have developed here promises to expediate and expand the development of future electrogenetic systems for a myriad of applications.

\section{Methods and Materials}

\section{Chemicals and Reagents:}

Primers and DNA parts (gBlocks ${ }^{\mathrm{TM}}$ ) were synthesized by Integrated DNA Technologies. PCR reactions were carried out using Phusion High-Fidelity DNA Polymerase from Thermo Fisher. Blunt end ligations were performed using the Thermo Fisher CloneJET PCR Cloning Kit. Enzyme digestions were performed using high-fidelity restriction enzymes purchased from New England Biolabs (NEB). DNA ligations were performed using Promega T4 ligase. Redox inducers and other chemicals and materials were purchased from Merck, unless otherwise stated. BASIC linkers were obtained from Biolegio and magnetic DNA purification was done using AMPure XP magnetic DNA purification kits. All organic redox inducers were stored as stock solutions at $-20{ }^{\circ} \mathrm{C}$ : methyl viologen dichloride hydrate (100 mM in LB), pyocyanin (50 mM in DMSO), DHNA (1 M in DMSO), Riboflavin (1 $\mathrm{mM}$ in LB), hydrogen peroxide (1 M in LB).

\section{Bacterial Strains, Plasmids and Media:}

The $E$. coli strains used were DH5 $\alpha$ (fhuA2 lac $\Delta \mathrm{U} 169$ phoA glnV44 $\Phi 80$ ' lacZ $\Delta \mathrm{M} 15$ gyrA96 recA1 relA1 endA1 thi-1 hsdR17), purchased from NEB, and DJ901 ( $\Delta$ (argFlac)169 $\lambda$ - IN(rrnD-rrnE)1 rpsL179(strR) zjc-2205::Tn10kan $\Delta$ (soxS-soxR)566), purchased from the Coli Genetic Stock Center (CGSC). E. coli strains were cultivated in 
LB medium and LB-agar plates at $37^{\circ} \mathrm{C}$ with corresponding antibiotics at the following concentrations: kanamycin $(50 \mu \mathrm{g} / \mathrm{ml})$, ampicillin $(50 \mu \mathrm{g} / \mathrm{ml})$, chloramphenicol $(25$ $\mu \mathrm{g} / \mathrm{ml}$ ). All plasmids contain pMB1 origin of replication with either kanamycin or chloramphenicol resistance, and $\mathrm{mScarlet}$ dropout-cassette at the insertion site. For plate reader experiments shown in Supplementary Figure 8, cells were grown in M9 medium ( $1 \times$ M9 salts, $0.4 \%$ glucose, $\left.2 \mathrm{mM} \mathrm{MgSO}_{4}, 100 \mu \mathrm{M} \mathrm{CaCl}_{2}\right)$ supplemented with $0.2 \%$ casamino acids and $100 \mathrm{mM}$ MOPS, as described in (22).

\section{BASIC DNA Assembly:}

All part plasmids were assembled by performing blunt end ligations of gBlocks ${ }^{\mathrm{TM}}$ into pJET1.2/blunt vectors. All construct plasmids were assembled using BASIC (Biopart Assembly Standard for Idempotent Cloning) assembly(66). Parts are listed in Supplementary Table 3, Constructs in Supplementary Table 3 and BASIC linkers in Supplementary Table 4. Plasmid maps are available on GitHub (https://github.com/JLawrence96/ElectrogeneticsToolset/tree/DNA)

\section{Mutant Library Generation:}

The promoter library was constructed using PCR mutagenesis with Phusion HighFidelity DNA Polymerase. Divergent 5' phosphorylated primers carrying the mutations were used to amplify the whole plasmid and introducer the mutations using the standard Phusion DNA polymerase protocol from NEB. Mutagenesis primers are listed in Supplementary Table 5). Reverse mutagenesis primers were used to mutate the -35 site while forward mutagenesis primers were used to mutate the -10 site. The DNA product was purified using PCR clean-up with QIAquick® PCR Purification Kit, followed by DpnI digestion (NEB) to cut methylated template DNA. Plasmids were re-ligated using Promega T4 before heat-shock transformation into E. coli DH5 $\alpha$.

\section{Chemical Heat-shock E.coli Transformation:}

Competent cell stocks of E. coli DH5 $\alpha$ and DJ901 were prepared by the Inoue method(67) and stored at $-80^{\circ} \mathrm{C}$. Heat-shock transformation was performed by defrosting $50 \mu \mathrm{l}$ of competent DH5 $\alpha$ cells, and immediately adding this to a PCR tube containing $5 \mu \mathrm{l}$ of plasmid DNA. This mixture was incubated on ice for 20 min before transferring to a thermocycler for heat-shock. Heat-shock was performed with a protocol consisting of $20 \mathrm{~min}$ a $4{ }^{\circ} \mathrm{C}, 45 \mathrm{~s}$ at $42{ }^{\circ} \mathrm{C}$ and $2 \mathrm{~min}$ at $4{ }^{\circ} \mathrm{C}$. Following heatshock, $200 \mu \mathrm{L}$ of SOC broth pre-warmed to $37^{\circ} \mathrm{C}$ was added. Recovery of transformed culture was performed at $37^{\circ} \mathrm{C}$ for $1 \mathrm{hr}$ before $100 \mu \mathrm{L}$ was plated on LB-agar supplemented with the appropriate antibiotic. 


\section{Stationary Phase Measurements:}

Glycerol stock of strains containing the constructs and control plasmids were streaked on LB-agar antibiotic plates and incubated overnight at $37^{\circ} \mathrm{C}$. Plates were stored at $4^{\circ} \mathrm{C}$. Single colonies were picked from these plates and inoculated into $5 \mathrm{~mL} \mathrm{LB}$

supplemented with the appropriate antibiotic and incubated overnight at $37^{\circ} \mathrm{C} .2 \mu \mathrm{L}$ of overnight cultures were diluted in $198 \mu \mathrm{L}$ of LB + antibiotics with or without pyocyanin to an $\mathrm{OD}_{600}$ of approximately 0.1 . LB media containing pyocyanin was created by diluting pyocyanin stock solutions in LB so that diluted overnight cultures had the desired working concentration of pyocyanin (either 2.5 or $10 \mu \mathrm{M}$ ). These cultures were transferred to wells of a 96-well microplate (Costar) which was sealed with BreatheEasy® sealing membrane which was placed in a Synergy HT microplate reader (BioTek) and incubated at $37^{\circ} \mathrm{C}$ with orbital shaking at $1000 \mathrm{rpm}$ for $14 \mathrm{~h}$. After incubation, endpoint sfGFP fluorescence (excitation, $485 \mathrm{~nm}$; emission, $528 \mathrm{~nm}$; gain 40) and OD 600 measurements were taken with a Synergy HT microplate reader (BioTek). For measuring the upstream activity, RFP measurements (excitation, $590 \mathrm{~nm}$; emission, 645 nm; gain 70) were also taken. All measurements were taken from the bottom. Outputs were expressed in units of RPU. This was calculated by subtracting blank fluorescence and $\mathrm{OD}_{600}$ measurements from the data (which were recorded from measurements of the media condition without cells), before dividing fluorescence measurements by $\mathrm{OD}_{600}$ measurements and expressing them as a ratio relative to the same measurements recorded from a strain harbouring an RPU standard plasmid in LB medium. This plasmid contained the same fluorophore being expressed from a J23101 promoter with the same standardised design used for the other promoters in this study $(38,41)$ (Supplementary Table 3).

For plate reader experiments shown in Supplementary Figure 8, cells were treated identically except M9 medium (Bacterial Strains, Plasmids and Media) was used instead of LB medium.

\section{Response Function Measurements:}

Microplates were prepared identically to how they were prepared for stationary phase measurements, apart from overnight cultures were diluted in LB or M9 media + antibiotics containing different redox inducers to a range of different redox inducer concentrations. Response curves were calculated by a previously developed $\operatorname{method}(14)$ in which experimental data was fitted to a straight line for non-responsive constructs, to equation [1] for Uni-PsoxS and activator devices, and to equation [2] for inverter devices:

$$
\begin{aligned}
& y_{R F}=y_{0}+\left(y_{\max }-y_{0}\right) \frac{x^{n}}{K^{n}+x^{n}} \\
& y_{R F}=y_{0}-\left(y_{0}-y_{\text {min }}\right) \frac{x^{n}}{K^{n}+x^{n}}
\end{aligned}
$$

where $y_{R F}$ is the fitted response function. The fixed parameters were: $x$, the concentration of redox inducer; $y_{0}$, the RPU with no redox inducer added; $y_{\max }$, the 
largest achieved RPU value across all concentrations of redox inducer. The fitted parameters were: $K$, the sensitivity; $n$, the cooperativity.

Fitting was performed in MATLAB using a custom script utilising the fminsearch function. Response functions were fit by the least-squares method to minimise the Sum of Squared Estimate of Errors (SSE) between the data and the model, as detailed in equation [3]:

$$
S S E=\sum_{i=1}^{N}\left[y_{R F}\left(x_{i}\right)-y_{E x}\left(x_{i}\right)\right]^{2}
$$

where $y_{R F}\left(x_{i}\right)$ is the response function RPU value and $y_{E x}\left(x_{i}\right)$ is the experimental RPU value for a given concentration of redox inducer $x_{i}$, with $N$ being the total number of redox inducer concentrations tested.

The dynamic rage was calculated from experimental data, as detailed in equation [4] for Uni-PsoxS and activator devices, and to equation [5] for inverter devices:

$$
\begin{aligned}
& \text { DynR }=\frac{y_{0}}{y_{\min }} \\
& \text { DynR }=\frac{y_{\max }}{y_{0}}
\end{aligned}
$$

The $\mathrm{R}^{2}$ was also calculated for each model to determine the quality of the fit, as detailed in equation [6]:

$$
R^{2}=\frac{S S E}{\sum_{i=1}^{N}\left[y_{E x}\left(x_{i}\right)-\overline{y_{E x}\left(x_{l}\right)}\right]^{2}}
$$

All experimental and calculated values of response functions are listed in Supplementary Table 1. All Code is available on Github (https://github.com/JLawrence96/ElectrogeneticsToolset/tree/Code)

\section{Cyclic Voltammetry:}

Cyclic voltammetry was performed with a PalmSens EmStat3 Blue. A 20 mL glass vial was used as an electrochemical cell with a glassy carbon working electrode, platinum mesh counter electrode and a Ag/AgCl reference electrode (Supplementary Fig. 6a). Voltammograms were recorded from $5 \mathrm{~mL}$ of LB medium alone, or supplemented with either $1 \mathrm{mM}$ of methyl viologen, $100 \mu \mathrm{M}$ of pyocyanin or $1 \mathrm{mM}$ DHNA. The cell was heated to $37{ }^{\circ} \mathrm{C}$ using a hot plate. Three scans were performed for each sample between using a scan rate of $10 \mathrm{mV} \mathrm{s}^{-1}$. Scans were recorded both with and without purging. Purging was performed for 30 mins with $\mathrm{N}_{2}$ gas bubbled through a long needle, with said needle being placed in the headspace of the vial when the scan was recorded to maintain anoxic conditions. The third scan for each experiment was recorded, with the corresponding scan of LB medium over the same potential being subtracted from it. $\mathrm{E}_{\mathrm{m}}$ values were calculated from the purged condition from the potential lying equidistant 
between the oxidation and reduction peaks. Potentials were converted from $\mathrm{mV}$ vs $\mathrm{Ag} / \mathrm{AgCl}$ to $\mathrm{mV}$ vs SHE by addition of $200 \mathrm{mV}$.

\section{Bioelectrochemical Device Fabrication:}

The modular bioelectrochemical device used in this study consisted of a stack of acrylic blocks (Engineering \& Design Plastics Ltd, UK), polydimethylsiloxane (PDMS) gaskets, carbon paper electrodes (Fuel Cell Store, Texas, US), Nafion® 117 membrane and stainless-steel mesh electrical connectors (MeshDirect Ltd, UK).

From front to back, the stack was formed of: solid acrylic blocks (Supplementary Fig. 9a) serving as a front plate followed by a carbon paper disk working electrode (Supplementary Fig. 9e) with a stainless-steel mesh used as an electrical connector (Supplementary Fig. 9g) and a PDMS gasket (Supplementary Fig. 9b). Then, two acrylic blocks with cylindrical central cavity (Supplementary Fig. 9c-d) were placed next to each other forming the working chamber. A reference electrode was placed in the working chamber by a vertical port present in one of the acrylic blocks. The working chamber was followed by a disk of Nafion $® 117$ membrane (Supplementary Fig. 9f) sandwiched between two PDMS gasket (Supplementary Fig. 9f). The device continued with an acrylic block with a cylindrical central cavity (Supplementary Fig. 9b) forming the cathodic chamber followed by a PDMS gasket (Supplementary Fig. 9f), a carbon paper disk counter electrode (Supplementary Fig. 9e) and a stainless-steel mesh used as an electrical connector (Supplementary Fig. 9g). The device was then completed with a solid acrylic block for the back plate (Supplementary Fig. 9a). All those components were fastened together with fours stainless-steel screws. The complete device is shown in Fig. 6a and Supplementary Fig. 9h. Magnetic stir bars providing mixing were placed in each chamber and the device was placed atop a multi-plate stirrer (Svelp Scientific, Italy), with rubber stoppers being used to plug any cavities during experiments.

\section{Electrochemical Induction of Gene Expression:}

Overnight cultures were diluted in $\mathrm{LB}+$ chloramphenicol to a final $\mathrm{OD}_{600}$ of 0.05 . Pyocyanin stock was added to cultures to achieve a final concentration of $10 \mu \mathrm{M} .30 \mathrm{~mL}$ of culture was loaded into each chamber of the bioelectrochemical device which was then sealed with rubber plugs, placed on a magnetic stirrer in an incubator set to $37^{\circ} \mathrm{C}$ and connected to a PalmSens EmStat3 Blue. Chronoamperometry was performed for 16 $\mathrm{hr}$ with an applied bias potential of $-500 \mathrm{mV}$ vs $\mathrm{Ag} / \mathrm{AgCl}(-300 \mathrm{mV}$ vs SHE) and a sampling rate of $1 \mathrm{~s}^{-1}$. Following this cultures from each chamber were visualised by confocal fluorescence microscopy and then concentrated 4-fold before measuring OD 600 using a X UV-visible spectrophotometer and fluorescence using an Edinburgh Instruments FS5 Spectrofluorometer (excitation, $485 \mathrm{~nm}$; excitation bandwidth, $1 \mathrm{~nm}$; emission, $528 \mathrm{~nm}$; emission bandwidth, $0.3 \mathrm{~nm}$ ). Output was measured by dividing fluorescence measurements by $\mathrm{OD}_{600}$ measurements obtained using a Varian Cary 50 Bio UV-vis spectrometer. 


\section{Confocal Fluorescence Microscopy:}

Live Inv106 (DH5 $\alpha$ ) cells that were grown in the working and counter chambers of the bioelectrochemical device were immobilised on agarose pads made of $1 \%$ low-melt agarose solution in water(68). Images were acquired using a Nikon Eclipse Ti widefield microscope with a Nikon objective lens (Plan APO, $100 \times / 1.45$ oil) and a Hamamatsu C11440, ORCA Flash 4.0 camera. Brightfield and fluorescence of sfGFP ( $\lambda_{\text {excitation }}=490$ $\mathrm{nm}$ ) images were taken. Images were processed using NIS Elements Viewer and Image J software.

\section{References}

1. S. Slomovic, K. Pardee, J. J. Collins, Synthetic biology devices for in vitro and in vivo diagnostics. Proc. Natl. Acad. Sci. U. S. A. 112, 14429-14435 (2015).

2. M. J. Smanski, H. Zhou, J. Claesen, B. Shen, M. A. Fischbach, C. A. Voigt, Synthetic biology to access and expand nature's chemical diversity. Nat. Rev. Microbiol. 14, 135-149 (2016).

3. C. Gilbert, T. Ellis, Biological Engineered Living Materials: Growing Functional Materials with Genetically Programmable Properties. ACS Synth. Biol. 8, 1-15 (2019).

4. Y. Liu, J. Li, T. Tschirhart, J. L. Terrell, E. Kim, C.-Y. Tsao, D. L. Kelly, W. E. Bentley, G. F. Payne, Connecting Biology to Electronics: Molecular Communication via Redox Modality. Adv. Healthc. Mater. 6, 1700789 (2017).

5. M. D. Engstrom, B. F. Pfleger, Transcription control engineering and applications in synthetic biology. Synth. Syst. Biotechnol. 2, 176-191 (2017).

6. C. A. Voigt, Genetic parts to program bacteria. Curr. Opin. Biotechnol. 17, 548-557 (2006).

7. L. Borghi, Inducible gene expression systems for plants. Methods Mol. Biol. 655, 65-75 (2010).

8. H. Redden, N. Morse, H. S. Alper, The synthetic biology toolbox for tuning gene expression in yeast. FEMS Yeast Res. 15, 1-10 (2015).

9. Kallunki, Barisic, Jäättelä, Liu, How to Choose the Right Inducible Gene Expression System for Mammalian Studies? Cells. 8, 796 (2019).

10. L. B. Andrews, A. A. K. Nielsen, C. A. Voigt, Cellular checkpoint control using programmable sequential logic. Science. 361, eaap8987 (2018).

11. B. Wang, M. Barahona, M. Buck, A modular cell-based biosensor using engineered genetic logic circuits to detect and integrate multiple environmental signals. Biosens. Bioelectron. 40, 368-376 (2013).

12. A. D. Silverman, U. Akova, K. K. Alam, M. C. Jewett, J. B. Lucks, Design and Optimization of a Cell-Free Atrazine Biosensor. ACS Synth. Biol. 9, 671-677 (2020). 
13. L. E. Dow, J. Fisher, K. P. O’Rourke, A. Muley, E. R. Kastenhuber, G. Livshits, D. F. Tschaharganeh, N. D. Socci, S. W. Lowe, Inducible in vivo genome editing with CRISPR-Cas9. Nat. Biotechnol. 33, 390-394 (2015).

14. A. J. Meyer, T. H. Segall-Shapiro, E. Glassey, J. Zhang, C. A. Voigt, Escherichia coli "Marionette" strains with 12 highly optimized small-molecule sensors. Nat. Chem. Biol. 15, 196-204 (2019).

15. M. A. Lalwani, S. S. Ip, C. Carrasco-López, C. Day, E. M. Zhao, H. Kawabe, J. L. Avalos, Optogenetic control of the lac operon for bacterial chemical and protein production. Nat. Chem. Biol. 17, 71-79 (2020).

16. N. A. Valdez-Cruz, L. Caspeta, N. O. Pérez, O. T. Ramírez, M. A. Trujillo-Roldán, Production of recombinant proteins in E. coli by the heat inducible expression system based on the phage lambda pL and/or pR promoters. Microb. Cell Factories 9, 1-16 (2010).

17. V. Ortner, C. Kaspar, C. Halter, L. Töllner, O. Mykhaylyk, J. Walzer, W. H. Günzburg, J. A. Dangerfield, C. Hohenadl, T. Czerny, Magnetic field-controlled gene expression in encapsulated cells. J. Control. Release. 158, 424-432 (2012).

18. T. J. Welch, D. H. Bartlett, Identification of a regulatory protein required for pressure-responsive gene expression in the deep-sea bacterium Photobacterium species strain SS9. Mol. Microbiol. 27, 977-985 (1998).

19. T. Yoshida, L. Qin, L. A. Egger, M. Inouye, Transcription regulation of ompF and ompC by a single transcription factor, OmpR. J. Biol. Chem. 281, 17114-17123 (2006).

20. J. Diray-Arce, A. Knowles, A. Suvorov, J. O’Brien, C. Hansen, S. M. Bybee, B. Gul, M. A. Khan, B. L. Nielsen, Identification and evolutionary characterization of saltresponsive transcription factors in the succulent halophyte Suaeda fruticosa. PLoS One. 14, e0222940 (2019).

21. C. S. Thiel, S. Hauschild, A. Huge, S. Tauber, B. A. Lauber, J. Polzer, K. Paulsen, H. Lier, F. Engelmann, B. Schmitz, A. Schütte, L. E. Layer, O. Ullrich, Dynamic gene expression response to altered gravity in human T cells. Sci. Rep. 7, 1-22 (2017).

22. T. Tschirhart, E. Kim, R. Mckay, H. Ueda, H. C. Wu, A. E. Pottash, A. Zargar, A. Negrete, J. Shiloach, G. F. Payne, W. E. Bentley, Electronic control of gene expression and cell behaviour in Escherichia coli through redox signalling. Nat. Commun. 8, 1-11 (2017).

23. D. Motabar, J. Li, G. F. Payne, W. E. Bentley, Mediated electrochemistry for redoxbased biological targeting: entangling sensing and actuation for maximizing information transfer. Curr. Opin. Biotechnol. 71, 137-144 (2021).

25. A. Hirose, A. Kouzuma, K. Watanabe, Towards development of electrogenetics using electrochemically active bacteria. Biotechnol. Adv. 37, 107351 (2019).

26. N. Bhokisham, E. VanArsdale, K. T. Stephens, P. Hauk, G. F. Payne, W. E. Bentley, A redox-based electrogenetic CRISPR system to connect with and control biological information networks. Nat. Commun. 11, 2427 (2020).

27. S. S. Yim, R. M. McBee, A. M. Song, Y. Huang, R. U. Sheth, H. H. Wang, Robust direct 
digital-to-biological data storage in living cells. Nat. Chem. Biol. 17, 246-253 (2021).

28. K. Krawczyk, S. Xue, P. Buchmann, G. Charpin-El-Hamri, P. Saxena, M.-D. Hussherr, J. Shao, H. Ye, M. Xie, M. Fussenegger, Electrogenetic cellular insulin release for real-time glycemic control in type 1 diabetic mice. Science. 368, 993-1001 (2020).

29. J. L. Terrell, T. Tschirhart, J. P. Jahnke, K. Stephens, Y. Liu, H. Dong, M. M. Hurley, M. Pozo, R. McKay, C. Y. Tsao, H.-C. Wu, G. Vora, G. F. Payne, D. N. Stratis-Cullum, W. E. Bentley, Bioelectronic control of a microbial community using surface-assembled electrogenetic cells to route signals. Nat. Nanotechnol. 16, 688-697 (2021).

30. E. R. Clifford, R. W. Bradley, L. T. Wey, J. M. Lawrence, X. Chen, C. J. Howe, J. Z. Zhang, Phenazines as model low-midpoint potential electron shuttles for photosynthetic bioelectrochemical systems. Chem. Sci. 12, 3328-3338 (2021).

31. J. A. N. Brophy, C. A. Voigt, Principles of genetic circuit design. Nat. Methods. 11, 508-520 (2014).

32. M. Storch, A. Casini, B. Mackrow, T. Fleming, H. Trewhitt, T. Ellis, G. S. Baldwin, BASIC: A New Biopart Assembly Standard for Idempotent Cloning Provides Accurate, Single-Tier DNA Assembly for Synthetic Biology. ACS Synth. Biol. 4, 781787 (2015).

33. K. Kobayashi, M. Fujikawa, T. Kozawa, Binding of Promoter DNA to SoxR Protein Decreases the Reduction Potential of the [2Fe-2S] Cluster. Biochemistry. 54, 334339 (2014).

34. A. K. Singh, J. H. Shin, K. L. Lee, J. A. Imlay, J. H. Roe, Comparative study of SoxR activation by redox-active compounds. Mol. Microbiol. 90, 983-996 (2013).

35. S. Watanabe, A. Kita, K. Kobayashi, K. Miki, Crystal structure of the [2Fe-2S] oxidative-stress sensor SoxR bound to DNA. Proc. Natl. Acad. Sci. U. S. A. 105, 4121-4126 (2008).

36. M. Chander, B. Demple, Functional analysis of SoxR residues affecting transduction of oxidative stress signals into gene expression. J. Biol. Chem. 279, 41603-41610 (2004).

37. H. M. Salis, E. A. Mirsky, C. A. Voigt, Automated design of synthetic ribosome binding sites to control protein expression. Nat. Biotechnol. 27, 946-950 (2009).

38. M. Storch, M. C. Haines, G. S. Baldwin, DNA-BOT: A low-cost, automated DNA assembly platform for synthetic biology. Synth. Biol. 5, ysaa010 (2020).

39. S. B. Carr, J. Beal, D. M. Densmore, Reducing DNA context dependence in bacterial promoters. PLoS One. 12, e0176013 (2017).

40. C. Lou, B. Stanton, Y. J. Chen, B. Munsky, C. A. Voigt, Ribozyme-based insulator parts buffer synthetic circuits from genetic context. Nat. Biotechnol. 30, 11371142 (2012).

41. J. R. Kelly, A. J. Rubin, J. H. Davis, C. M. Ajo-Franklin, J. Cumbers, M. J. Czar, K. de Mora, A. L. Glieberman, D. D. Monie, D. Endy, Measuring the activity of BioBrick promoters using an in vivo reference standard. J. Biol. Eng. 3, 4 (2009). 
42. M. Storch, A. Casini, B. Mackrow, T. Fleming, H. Trewhitt, T. Ellis, G. S. Baldwin, BASIC: A New Biopart Assembly Standard for Idempotent Cloning Provides Accurate, Single-Tier DNA Assembly for Synthetic Biology. ACS Synth. Biol. 4, 781787 (2015).

43. E. Martínez-Garćía, T. Aparicio, A. Goñi-Moreno, S. Fraile, V. De Lorenzo, SEVA 2.0: An update of the Standard European Vector Architecture for de-/re-construction of bacterial functionalities. Nucleic Acids Res. 43, D1183-D1189 (2015).

44. E. Hidalgo, V. Leautaud, B. Demple, The redox-regulated SoxR protein acts from a single DNA site as a repressor and an allosteric activator. EMBO J. 17, 2629-2636 (1998).

45. E. Hidalgo, B. Demple, Spacing of promoter elements regulates the basal expression of the soxS gene and converts SoxR from a transcriptional activator into a repressor carboxy-terminal domains of the RNAP $\alpha$ subunits. Activ. EMBO J. 16, 1056-1065 (1997).

46. Y. Chen, J. M. L. Ho, D. L. Shis, C. Gupta, J. Long, D. S. Wagner, W. Ott, K. Josić, M. R. Bennett, Tuning the dynamic range of bacterial promoters regulated by ligandinducible transcription factors. Nat. Commun. 9, 1-8 (2018).

47. G. Urtecho, A. D. Tripp, K. D. Insigne, H. Kim, S. Kosuri, Systematic Dissection of Sequence Elements Controlling 670 Promoters Using a Genomically Encoded Multiplexed Reporter Assay in Escherichia coli. Biochemistry. 58, 1539-1551 (2019).

48. B. P. Anton, E. A. Raleigh, Complete genome sequence of NEB 5-alpha, a derivative of Escherichia coli K-12 DH5a. Genome Announc. 4, e01245-16 (2016).

49. J. T. Greenberg, P. Monach, J. H. Chou, P. D. Josephy, B. Demple, Positive control of a global antioxidant defense regulon activated by superoxide-generating agents in Escherichia coli. Proc. Natl. Acad. Sci. U. S. A. 87, 6181-6185 (1990).

50. A. Zhang, J. L. Rosner, R. G. Martin, Transcriptional activation by MarA, SoxS and Rob of two tolC promoters using one binding site: a complex promoter configuration for tolC in Escherichia coli. Mol. Microbiol. 69, 1450-1455 (2008).

51. A. Gutiérrez-Preciado, A. G. Torres, E. Merino, H. R. Bonomi, F. A. Goldbaum, V. A. García-Angulo, Extensive identification of bacterial riboflavin transporters and their distribution across bacterial species. PLoS One. 10, e0126124. (2015).

52. M. Zheng, X. Wang, L. J. Templeton, D. R. Smulski, R. A. Larossa, G. Storz, DNA Microarray-Mediated Transcriptional Profiling of the Escherichia coli Response to Hydrogen Peroxide. J. Bacteriol. 183, 4562-4570 (2001).

53. M. Fujikawa, K. Kobayashi, T. Kozawa, Direct Oxidation of the [2Fe-2S] Cluster in SoxR Protein by Superoxide: Distinct Differential Sensitivity to Superoxidemediated Signal Transduction. J. Biol. Chem. 287, 35702-35708 (2012).

54. M. Gu, J. A. Imlay, The SoxRS response of Escherichia coli is directly activated by redox-cycling drugs rather than by superoxide. Mol. Microbiol. 79, 1136 (2011).

55. Y. Huang, H. Zhan, P. Bhatt, S. Chen, Paraquat Degradation From Contaminated Environments: Current Achievements and Perspectives. Front. Microbiol. 10, 
1754 (2019).

56. D. J. Hassett, L. Charniga, K. Bean, D. E. Ohman, M. S. Cohen, Response of Pseudomonas aeruginosa to pyocyanin: mechanisms of resistance, antioxidant defenses, and demonstration of a manganese-cofactored superoxide dismutase. Infect. Immun. 60, 328 (1992).

57. M. Chen, X. Ma, X. Chen, M. Jiang, H. Song, Z. Guo, Identification of a hotdog fold thioesterase involved in the biosynthesis of menaquinone in Escherichia coli. J. Bacteriol. 195, 2768-2775 (2013).

58. J. Feng, Y. Qian, Z. Wang, X. Wang, S. Xu, K. Chen, P. Ouyang, Enhancing the performance of Escherichia coli-inoculated microbial fuel cells by introduction of the phenazine-1-carboxylic acid pathway. J. Biotechnol. 275, 1-6 (2018).

59. M. W. Gander, J. D. Vrana, W. E. Voje, J. M. Carothers, E. Klavins, Digital logic circuits in yeast with CRISPR-dCas9 NOR gates. Nat. Commun. 8, 1-11 (2017).

60. M. Zhou, M. Chi, J. Luo, H. He, T. Jin, An overview of electrode materials in microbial fuel cells. J. Power Sources. 196, 4427-4435 (2011).

61. S. Guo, R. Liu, W. Wang, H. Hu, Z. Li, X. Zhang, Designing an Artificial Pathway for the Biosynthesis of a Novel Phenazine N -Oxide in Pseudomonas chlororaphis HT66. ACS Synth. Biol. 9, 883-892 (2020).

62. D. Li, L. Zhang, W. Gao, J. Meng, Y. Guan, J. Liang, X. Shen, Electrochemical degradation of chloramphenicol using Ti-based SnO2-Sb-Ni electrode. Water Sci. Technol. 84, 1 (2021).

63. L. E. P. Dietrich, T. K. Teal, A. Price-Whelan, D. K. Newman, Redox-active antibiotics control gene expression and community behavior in divergent bacteria. Science. 321, 1203-1206 (2008).

64. H. He, F. Van Breusegem, A. Mhamdi, Redox-dependent control of nuclear transcription in plants. J. Exp. Bot. 69, 3359-3372 (2018).

65. R. Brigelius-Flohé, L. Flohé, Basic principles and emerging concepts in the redox control of transcription factors. Antioxidants Redox Signal. 15, 2335-2381 (2011).

66. M. Storch, A. Casini, B. Mackrow, T. Ellis, G. S. Baldwin, BASIC: A Simple and Accurate Modular DNA Assembly Method. Methods Mol. Biol. 1472, 79-91 (2017).

67. H. Inoue, H. Nojima, H. Okayama, High efficiency transformation of Escherichia coli with plasmids. Gene. 96, 23-28 (1990).

68. S. O. Skinner, L. A. Sepúlveda, H. Xu, I. Golding, Measuring mRNA copy number in individual Escherichia coli cells using single-molecule fluorescent in situ hybridization. Nat. Protoc. 8, 1100-1113 (2013).

Acknowledgements: We thank Prof. Richard Kitney, Prof. Tom Ellis, Dr. Robert Bradley and Dr. Ari Dwijayanti for helpful discussions. Funding: This work was supported by the Biotechnology and Biological Sciences Research Council (BB/M011194/1 for JML, BB/R011923/1 for JZ), the Italian Ministry of University and Research 
(SIR2014/RBSI14JKU3 for PB), the Cambridge Trust (for LTW) and the Engineering and Physical Sciences Research Council (EP/S001859/1 for MS) Author contributions: J.M.L, Y.Y \& R.L-A designed the study. J.M.L \& Y.Y performed the experiments. Pixcell iGEM Team performed preliminary studies. P.B. constructed the bioelectrochemical device. A.S. assisted with plate reader experiments. M.S assisted with BASIC assembly. L.T.W. performed the fluorescence microscopy. J.M.L developed the code, analysed the data and wrote the manuscript with input from all authors. Competing interests: The authors declare that they have no competing interests. Data and materials

availability: All data needed to evaluate the conclusions in the paper are present in the paper and/or the Supplementary Materials. All input data, code and plasmid maps from this study are available on GitHub here:

https://github.com/JLawrence96/ElectrogeneticsToolset. 